PoS $\quad \begin{aligned} & \text { PROCEEDINGS } \\ & \text { OF SCIENCE }\end{aligned}$

\title{
Recent status of neutrino phenomenology
}

\section{OSAMU YASUDA*}

Department of Physics, Tokyo Metropolitan University

Hachioji, Tokyo 192-0397, Japan

E-mail: vasuda AT phys.se.tmu.ac.7p

Recent status of research on neutrino phenomenology is briefly reviewed.

XIV International Conference on Heavy Quarks and Leptons (HQL2018)

May 27- June 1, 2018

Yamagata Terrsa, Yamagata,Japan

${ }^{*}$ Speaker. 


\section{Introduction}

Since the discovery of atmospheric neutrino oscillation by Superkamiokande [四] and solar neutrino oscillation by SNO [వ] and KamLAND [B]], we know that neutrinos have masses and mixings. Just like the mass $\left((d, s, b)^{T}\right)$ and flavor $\left(\left(d^{\prime}, s^{\prime}, b^{\prime}\right)^{T}\right)$ eigenstates of the down quark sector are related by

$$
\left(\begin{array}{c}
d^{\prime} \\
s^{\prime} \\
b^{\prime}
\end{array}\right)=V_{C K M}\left(\begin{array}{c}
d \\
s \\
b
\end{array}\right)
$$

the mass $\left(\left(v_{1}, v_{2}, v_{3}\right)^{T}\right)$ and flavor $\left(\left(v_{e}, v_{\mu}, v_{\tau}\right)^{T}\right)$ eigenstates of the neutrino sector are related by

$$
\left(\begin{array}{l}
v_{e} \\
v_{\mu} \\
v_{\tau}
\end{array}\right)=U\left(\begin{array}{l}
v_{1} \\
v_{2} \\
v_{3}
\end{array}\right)
$$

where

$$
U=\left(\begin{array}{ccc}
c_{12} c_{13} & s_{12} c_{13} & s_{13} e^{-i \delta} \\
-s_{12} c_{23}-c_{12} s_{23} s_{13} e^{i \delta} & c_{12} c_{23}-s_{12} s_{23} s_{13} e^{i \delta} & s_{23} c_{13} \\
s_{12} s_{23}-c_{12} c_{23} s_{13} e^{i \delta} & -c_{12} s_{23}-s_{12} c_{23} s_{13} e^{i \delta} & c_{23} c_{13}
\end{array}\right)
$$

is the $3 \times 3$ unitary neutrino mixing matrix [ [4, []], $\theta_{12}, \theta_{23}, \theta_{13}$ are three mixing angles, $\delta$ is a CP phase, and $c_{j k} \equiv \cos \theta_{j k}, s_{j k} \equiv \sin \theta_{j k}$.

Recent results from cosmology [6] suggest that $\sum_{j} m_{j}<\mathscr{O}(0.1) \mathrm{eV}$ (95\% C.L.), and the fact that neutrino mass is much smaller than those of quarks and charged leptons is a mystery. The reason that neutrinos have relevance to this conference is because of the seesaw mechanism [ $\square$, 圆, 9, [0, [] which may solve this mystery. If the heavy Majorana mass $M$ is much heavier than the Dirac mass $m$, then the absolute value of the smaller mass eigenvalue of the mass matrix

$$
\left(\begin{array}{ll}
0 & m \\
m & M
\end{array}\right)
$$

is approximately equal to $\mathrm{m}^{2} / M$. Assuming that the largest neutrino mass can be approximately evaluated as $\left(\Delta m_{\mathrm{atm}}^{2}\right)^{1 / 2} \simeq 0.05 \mathrm{eV}$, that the Dirac mass is roughly equal to $m \sim \mathscr{O}(1) \mathrm{GeV}$, and that the smaller eigenvalue of the matrix $(\mathbb{L 2})$ is approximately equal to the largest neutrino mass $m_{3}$, then the heavy Majorana mass is estimated to be $M \sim \mathscr{O}\left(10^{10}\right) \mathrm{GeV}$. Such a high mass scale indicates that small neutrino mass may give a hint of physics beyond the Standard Model.

\section{Three flavor neutrino oscillation}

In the standard framework of three massive neutrinos, the parameters which describe neutrino oscillation phenomena are the three mixing angles $\theta_{12}, \theta_{23}, \theta_{13}$ and the CP phase $\delta$, which appear in (메), and two mass squared differences $\Delta m_{21}^{2}, \Delta m_{31}^{2}$, where $\Delta m_{j k}^{2} \equiv m_{j}^{2}-m_{k}^{2}$. The sets of parameters $\left(\left|\Delta m_{31}^{2}\right|, \theta_{23}\right)$ and $\left(\Delta m_{21}^{2}, \theta_{12}\right)$ were determined by the experiments of atmospheric and 
accelerator neutrinos, and by those of solar and long baseline reactor neutrinos, respectively [焑]. On the other hand, the value of $\theta_{13}$ was determined by the accelerator neutrino experiments, T2K [U2], MINOS [14] and NOvA [15]], and by the reactor neutrino experiments, Double-CHOOZ [ए6], Daya

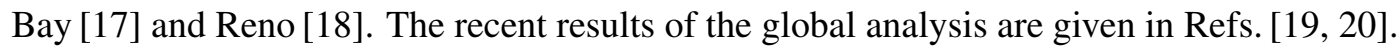

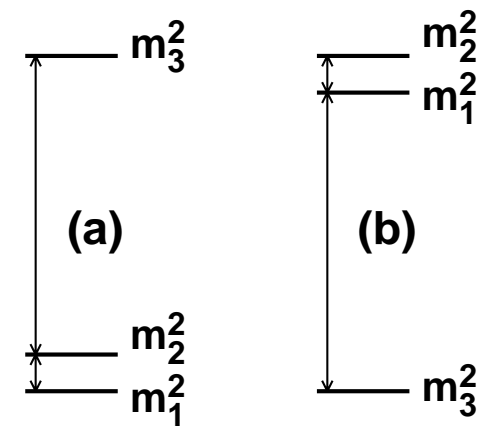

Figure 1: Two mass patterns: (a) the normal hierarchy $\left(\Delta m_{31}^{2}>0\right)$, (b) the inverted hierarchy $\left(\Delta m_{31}^{2}<0\right)$.

The quantities which are not yet determined are the pattern of mass hierarchy (the two mass

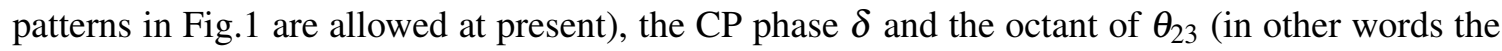
sign of $\theta_{23}-45^{\circ}$ ). The next things to do are to determine the pattern of mass hierarchy, the octant of $\theta_{23}$ and the CP phase $\delta$. These tasks are expected to be carried out in the future long baseline

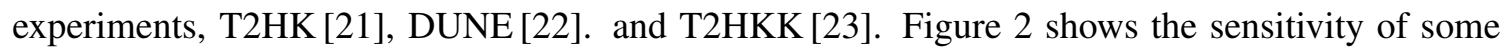
of these experiments together with atmospheric neutrino measurements at the Hyperkamiokande (HK) experiment [24] to the unknown quantities.

\section{Non-oscillation experiments}

While neutrino oscillation gives us the value of the mass squared differences $\Delta m_{j k}^{2}(j, k=$ $1,2,3)$, the mixing angles and the CP phase, complementary information can be obtained by other experiments, such as neutrinoless double beta decays, direct measurements by tritium decays and cosmology.

\subsection{Neutrinoless double beta decay}

If we assume the existence of Majorana mass terms, which violate the lepton number, then the process of a neutrinoless double beta decay $(A, Z) \rightarrow(A, Z+2)+e^{-}+e^{-}$becomes possible. The amplitude of the neutrinoless double beta decay process is proportional to the effective Majorana mass

$$
m_{e e}=\left|\sum_{j}\left(U_{e j}\right)^{2} e^{i \phi_{j}} m_{j}\right|,
$$

where $\phi_{j}$ stands for the Majorana phases. In the case of the inverted hierarchy, the effective Majo-

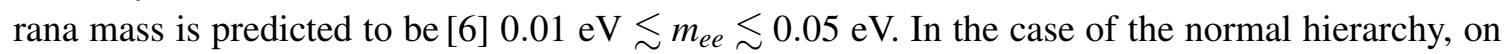
the other hand, there can be cancellation between the contributions among $m_{j}(j=1,2,3)$ and the 

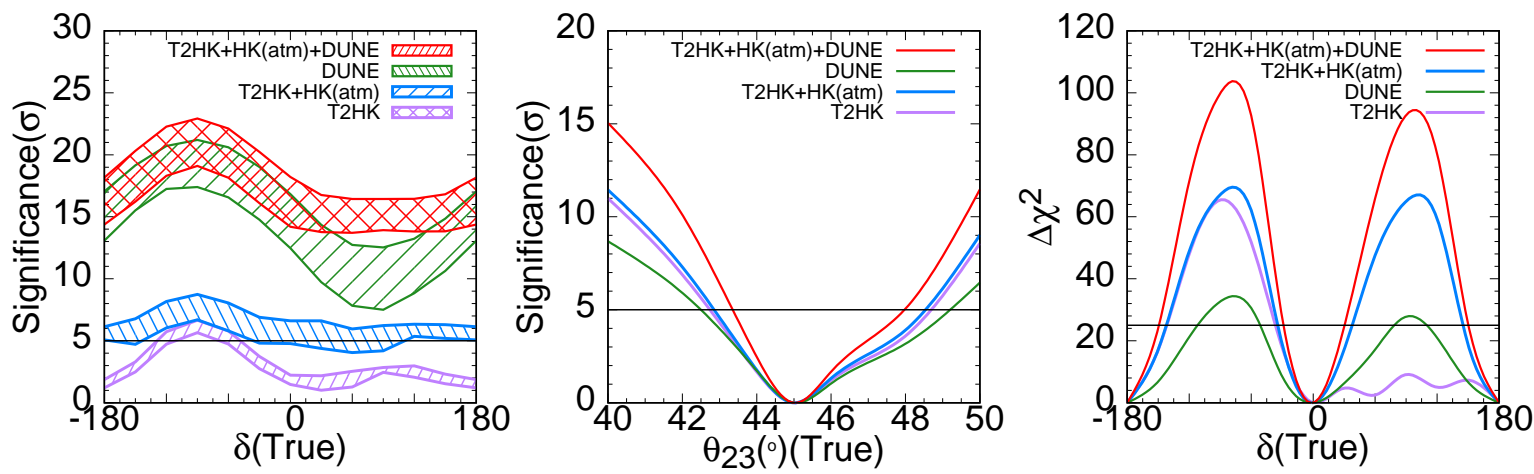

Figure 2: Sensitivity of the future experiments and their combinations to the unknown quantities in the case of normal hierarchy [[3]]. Left panel: Significance of mass hierarchy as a function of the true value of the CP phase $\delta$. The DUNE experiment, the combination of the T2HK experiment and the atmospheric neutrino observation at $\mathrm{HK}$, and the combination of all these can determine the mass hierarchy irrespective of the value of the CP phase $\delta$. Middle panel: Significance of octant as a function of the true value of $\theta_{23}$. If $\theta_{23} \lesssim 42^{\circ}$ or $\theta_{23} \gtrsim 49^{\circ}$, then T2HK, DUNE, the combination of T2HK and the atmospheric neutrino observation at $\mathrm{HK}$, and the combination of all these can distinguish whether $\theta_{23}>45^{\circ}$ or $\theta_{23}<45^{\circ}$. Right panel: $\Delta \chi^{2}=\{\text { significance of CP violation }(\sigma)\}^{2}$ as a function of the true value of the CP phase $\delta$. Unless $\delta$ is close to 0 or $180^{\circ}$, DUNE, the combination of T2HK and atmospheric neutrino observation at $\mathrm{HK}$ and the combination of all these can exclude the hypothesis $\delta=0$ or $\delta=180^{\circ}$. The horizontal straight line in each panel stands for $5 \sigma$ which corresponds to discovery.

prediction for the effective Majorana mass is [ [ $]$ ] $0 \mathrm{eV} \leq m_{e e} \lesssim 0.004 \mathrm{eV}$. The current best limit is given by KamLAND-Zen [25]]: $m_{e e}<(0.061-0.165) \mathrm{eV}$, where the uncertainty comes from that of the nuclear matrix elements which are required to estimate the value of $m_{e e}$.

\subsection{Direct measurement}

While neutrinoless double beta decays occur only when the majorana mass term exists, one can always perform a direct measurement of neutrino mass by looking at the energy spectrum of electrons near the end point in tritium decays. In this case the effective neutrino mass is given by

$$
m_{\beta}=\left(\sum_{j}\left|U_{e j}\right|^{2} m_{j}^{2}\right)^{1 / 2} .
$$

In the case of the normal (inverted) hierarchy, the prediction for the effective Majorana mass is [6] $0.004 \mathrm{eV}(0.03 \mathrm{eV}) \lesssim m_{\beta} \lesssim 0.01 \mathrm{eV}(0.06 \mathrm{eV})$, respectively. The present upper bound is obtained by the Troitsk experiment [26]: $m_{\beta}<2.05 \mathrm{eV}$ (at 95\% C.L.). The KATRIN experiment [27], which has just started, is expected to reach $m_{\beta} \sim 0.20 \mathrm{eV}$.

\subsection{Cosmology}

The constraint on $\sum_{j} m_{j}$ can be obtained from cosmology, and the most stringent one [RE] 


\begin{tabular}{|l|l|l|}
\hline Scenario & $\begin{array}{l}\text { Experimental } \\
\text { indication }\end{array}$ & $\begin{array}{l}\text { Phenomenological } \\
\text { constraint on the } \\
\text { relative deviation }\end{array}$ \\
\hline \hline Light sterile $v$ [B2] & Maybe & $O(10 \%)$ \\
\hline $\begin{array}{l}\text { NSI in propaga- } \\
\text { tion [B3], [34, [3]] }\end{array}$ & Maybe & $\begin{array}{l}\left.O(100 \%) \text { (for } v_{e}, v_{\tau}\right) \\
O(1 \%) \quad\left(\text { for } v_{\mu}\right)\end{array}$ \\
\hline $\begin{array}{l}\text { NSI at production / } \\
\text { detection [B]] }\end{array}$ & None & $O(1 \%)$ \\
\hline $\begin{array}{l}\text { Unitarity violation } \\
\text { due to } v_{R} \text { [B]] }\end{array}$ & None & $O(0.1 \%)$ \\
\hline
\end{tabular}

Table 1: Various scenarios beyond the standard model with massive three neutrinos.

$\sum_{j} m_{j}<0.14 \mathrm{eV}$ (95\% C.L.) is obtained by the Ly $\alpha$-forest power spectrum from the Baryon Oscillation Spectroscopic Survey and from the VLT/XSHOOTER legacy survey in the $\Lambda$ CDM $v$ model.

\section{New physics beyond the Standard Model with massive three neutrinos}

The future long baseline experiments [2], 22], 22] mentioned above have high statistics and, as in the case of B factories [ $[29,10]$, such high precision measurements will enable us to search for deviation from the standard three-flavor oscillations (see, e.g., Ref. [B]] ). Scenarios which have been discussed as candidates for deviation from the standard three-flavor framework include light sterile neutrinos [B2], the Non-Standard flavor-dependent Interaction (NSI) in neutrino propagation [33], [34, [35], NSI at production and/or detection [36], and unitarity violation due to right handed neutrinos [37]. As indicated in Table $\mathbb{W}$, the first two scenarios offer stronger phenomenological motivation because (i) there are experimental hints which may suggest these scenarios, (ii) the deviation of the oscillation probability for these scenarios could be potentially larger than those for the other scenarios and therefore it is encouraging for experimentalists to look for the effects of these scenarios.

\subsection{Light sterile neutrinos}

The anomaly which was announced by the LSND group [B8] would imply mass squared difference of $\mathscr{O}(1) \mathrm{eV}^{2}$ if it is interpreted as a phenomenon due to neutrino oscillation $\bar{v}_{\mu} \rightarrow \bar{v}_{e}$. The standard three flavor scheme has only two independent mass squared differences, i.e., $\Delta m_{21}^{2} \simeq$ $8 \times 10^{-5} \mathrm{eV}^{2}\left(\left|\Delta m_{31}^{2}\right| \simeq 2.4 \times 10^{-3} \mathrm{eV}^{2}\right)$ for the solar (atmospheric) neutrino oscillation. To accommodate a neutrino oscillation scheme to the LSND anomaly, therefore, an extra state should be introduced. This extra state should be sterile neutrino, which is a singlet with respect to the gauge group of the Standard Model, because the number of weakly interacting light neutrinos should be three from the LEP data [目]. To test the LSND anomaly, the MiniBooNE experiment has been performed, and their recent results [B]] seem to be statistically significant, although their allowed region seems to be incompatible with that of the negative results in the disappearance [40] and appearance [4]] channels. On the other hand, the flux of the reactor neutrino was recalculated in Refs. [42], [43] and it was claimed that the normalization is shifted by about $+3 \%$ on average. If 
their claim on the reactor neutrino flux is correct, then neutrino oscillation with $\Delta m^{2} \gtrsim 1 \mathrm{eV}^{2}$ may be concluded from a re-analysis of 19 reactor neutrino results at short baselines [44]. This is called reactor anomaly. Furthermore, it was pointed out in Ref. [45] that the measured and predicted ${ }^{71} \mathrm{Ge}$ production rates differ in the Gallium radioactive source experiments GALLEX and SAGE, and this is called Gallium anomaly. The anomalies of LSND, reactor and Gallium constitute the main motivation to study sterile neutrino oscillations.

\subsection{Non-standard interactions in propagation}

If we have a flavor-dependent neutrino non-standard interaction (NSI) in neutrino propagation:

$$
\mathscr{L}_{\mathrm{NSI}}=-2 \sqrt{2} \varepsilon_{\alpha \beta}^{f f^{\prime} P} G_{F}\left(\bar{v}_{\alpha L} \gamma_{\mu} v_{\beta L}\right)\left(\bar{f}_{P} \gamma^{\mu} f_{P}^{\prime}\right),
$$

where $f_{P}$ and $f_{P}^{\prime}$ are the fermions with chirality $P, \varepsilon_{\alpha \beta}^{f f^{\prime} P}$ is a dimensionless constant normalized in terms of the Fermi coupling constant $G_{F}$, then the matter potential in the flavor basis is modified as

$$
A\left(\begin{array}{ccc}
1+\varepsilon_{e e} & \varepsilon_{e \mu} & \varepsilon_{e \tau} \\
\varepsilon_{\mu e} & \varepsilon_{\mu \mu} & \varepsilon_{\mu \tau} \\
\varepsilon_{\tau e} & \varepsilon_{\tau \mu} & \varepsilon_{\tau \tau}
\end{array}\right)
$$

where $A \equiv \sqrt{2} G_{F} N_{e}$ stands for the matter effect, $\varepsilon_{\alpha \beta}$ is defined by $\varepsilon_{\alpha \beta} \equiv \sum_{f=e, u, d}\left(N_{f} / N_{e}\right) \varepsilon_{\alpha \beta}^{f}$ and $N_{f}(f=e, u, d)$ stands for number densities of fermions $f$. Here we defined the new NSI parameters as $\varepsilon_{\alpha \beta}^{f} \equiv \varepsilon_{\alpha \beta}^{f f L}+\varepsilon_{\alpha \beta}^{f f R}$ since the matter effect is sensitive only to the coherent scattering and only to the vector part in the interaction. In the case of the solar neutrino analysis [46], since the ratio of the density of protons to that of neutrons varies along the neutrino path, $\varepsilon_{\alpha \beta}^{f}(f=e, u, d)$ is assumed to be non-zero only for one particular fermion $f$ for simplicity. ${ }^{1}$ Furthermore, to discuss the effect of NSI on solar neutrinos, because solar neutrinos are approximately driven by one mass squared difference, $3 \times 3$ Hamiltonian is reduced to the following effective $2 \times 2$ Hamiltonian:

$$
H^{\mathrm{eff}}=\frac{\Delta m_{21}^{2}}{4 E}\left(\begin{array}{cc}
-\cos 2 \theta_{12} & \sin 2 \theta_{12} \\
\sin 2 \theta_{12} & \cos 2 \theta_{12}
\end{array}\right)+\left(\begin{array}{cc}
c_{13}^{2} A & 0 \\
0 & 0
\end{array}\right)+A \sum_{f=e, u, d} \frac{N_{f}}{N_{e}}\left(\begin{array}{cc}
-\varepsilon_{D}^{f} & \varepsilon_{N}^{f} \\
\varepsilon_{N}^{f *} & \varepsilon_{D}^{f}
\end{array}\right),
$$

where $\varepsilon_{D}^{f}$ and $\varepsilon_{N}^{f}$ are linear combinations of the standard NSI parameters:

$$
\begin{aligned}
\varepsilon_{D}^{f}= & c_{13} s_{13} \operatorname{Re}\left[e^{i \delta_{\mathrm{CP}}}\left(s_{23} \varepsilon_{e \mu}^{f}+c_{23} \varepsilon_{e \tau}^{f}\right)\right]-\left(1+s_{13}^{2}\right) c_{23} s_{23} \operatorname{Re}\left[\varepsilon_{\mu \tau}^{f}\right]-\frac{c_{13}^{2}}{2}\left(\varepsilon_{e e}^{f}-\varepsilon_{\mu \mu}^{f}\right) \\
& +\frac{s_{23}^{2}-s_{13}^{2} c_{23}^{2}}{2}\left(\varepsilon_{\tau \tau}^{f}-\varepsilon_{\mu \mu}^{f}\right) \\
\varepsilon_{N}^{f}= & c_{13}\left(c_{23} \varepsilon_{e \mu}^{f}-s_{23} \varepsilon_{e \tau}^{f}\right)+s_{13} e^{-i \delta_{\mathrm{CP}}}\left[s_{23}^{2} \varepsilon_{\mu \tau}^{f}-c_{23}^{2} \varepsilon_{\mu \tau}^{f *}+c_{23} s_{23}\left(\varepsilon_{\tau \tau}^{f}-\varepsilon_{\mu \mu}^{f}\right)\right] .
\end{aligned}
$$

It has been pointed out that the value of $\Delta m_{21}^{2}$ inferred from the solar neutrino data and that from the KamLAND experiment have a tension at $2 \sigma$, and the result of Ref. [46] shows that a nonvanishing value of $\left(\varepsilon_{D}^{f}, \varepsilon_{N}^{f}\right)$ solves this tension. This fact gives a motivation to take NSI in propagation seriously. $^{2}$

\footnotetext{
${ }^{1}$ The case of $f=e$ has not been analyzed because of the complication in which the NSI $\varepsilon_{\alpha \beta}^{e}$ would also affect the rate of the interactions between neutrinos and electrons at detection.

${ }^{2}$ It has been pointed out that a sterile neutrino scheme with the mass squared difference $\mathscr{O}\left(10^{-5} \mathrm{eV}^{2}\right)$ also solves the tension [47].
} 


\subsection{Non-standard interactions at production and detection}

A charged current flavor-dependent non-standard interaction $\varepsilon_{\alpha \beta} G_{F}\left(\bar{v}_{\alpha} \gamma_{\mu} \ell_{\beta}\right)\left(\bar{f} \gamma^{\mu} f^{\prime}\right)$ has been also discussed [36]. Here $\varepsilon_{\alpha \beta}(\alpha, \beta=e, \mu, \tau)$ is the coefficient of the non-standard interaction, normalized in terms of $G_{F}, v_{\alpha}$ and $\ell_{\alpha}(=e, \mu, \tau)$ are neutrinos and charged leptons of flavor $\alpha$, and $f$ and $f^{\prime}$ stand for fermions (the only relevant ones are electrons, $u$ and $d$ quarks). This interaction predicts the existence of exotic reactions such as $\pi^{+} \rightarrow \mu^{+}+v_{e}$ or $v_{\mu}+n \rightarrow p+\tau^{-}$in the process of production and detection of neutrinos. Ref. [48] studied the bounds on $\varepsilon_{\alpha \beta}$ (the coefficient of both charged and neutral current non-standard interactions) by various experiments and typically the bounds are strong for the charged current NSI: $\left|\varepsilon_{\alpha \beta}\right|<\mathscr{O}\left(10^{-2}\right)$. Sensitivity of various neutrino experiments to $\varepsilon_{\alpha \beta}$ has been investigated by many people [49, [0]].

\subsection{Violation of unitarity}

It is known [B]] that in generic see-saw models the kinetic term gets modified after integrating out the right handed neutrino and unitarity is expected to be violated. In the case of the so-called minimal unitarity violation, in which only three light neutrinos are involved and sources of unitarity violation are assumed to appear only in the neutrino sector, unitarity violation is strongly constrained. Its constraint mostly comes from the bounds of rare decays of charged leptons, and deviation from unitarity is at most of $\mathscr{O}(0.1 \%)[5]$.

\section{Summary}

In the standard framework of three massive neutrinos, all the three mixing angles have been determined, and the remaining quantities to be measured are the sign of $\Delta m_{31}^{2}$, the sign of $\theta_{23}-45^{\circ}$, and the $\mathrm{CP}$ phase $\delta$. These quantities are expected to be determined in the future experiments. In these future experiments, we can probe deviation from the standard three flavor framework, such as light sterile neutrino scenarios, non-standard interactions in propagation and those at production and detection, and unitarity violation. Some of these scenarios may account for the experimental anomalies, and further experiments are needed to confirm or refute these scenarios.

\section{Acknowledgments}

I would like to thank the organizers for invitation and hospitality during the workshop. This research was partly supported by a Grant-in-Aid for Scientific Research of the Ministry of Education, Science and Culture, under Grant No. 18K03653.

\section{References}

[1] Y. Fukuda et al. [Super-Kamiokande Collaboration], Phys. Rev. Lett. 81 (1998) 1562 doi:10.1103/PhysRevLett.81.1562 [hep-ex/9807003].

[2] Q. R. Ahmad et al. [SNO Collaboration], Phys. Rev. Lett. 89 (2002) 011301 doi:10.1103/PhysRevLett.89.011301 [nucl-ex/0204008].

[3] K. Eguchi et al. [KamLAND Collaboration], Phys. Rev. Lett. 90 (2003) 021802 doi:10.1103/PhysRevLett.90.021802 [hep-ex/0212021]. 
[4] B. Pontecorvo, Sov. Phys. JETP 6 (1957) 429 [Zh. Eksp. Teor. Fiz. 33 (1957) 549].

[5] Z. Maki, M. Nakagawa and S. Sakata, Prog. Theor. Phys. 28 (1962) 870. doi:10.1143/PTP.28.870

[6] M. Tanabashi et al. [Particle Data Group Collaboration], Phys. Rev. D 98 (2018) 030001.

[7] P. Minkowski, Phys. Lett. 67B (1977) 421. doi:10.1016/0370-2693(77)90435-X

[8] T. Yanagida, Conf. Proc. C 7902131 (1979) 95.

[9] M. Gell-Mann, P. Ramond and R. Slansky, Conf. Proc. C 790927 (1979) 315 [arXiv:1306.4669 [hep-th]].

[10] R. N. Mohapatra and G. Senjanovic, Phys. Rev. Lett. 44 (1980) 912. doi:10.1103/PhysRevLett.44.912

[11] J. Schechter and J. W. F. Valle, Phys. Rev. D 22 (1980) 2227. doi:10.1103/PhysRevD.22.2227

[12] K. Abe et al. [T2K Collaboration], Phys. Rev. Lett. 107 (2011) 041801 [arXiv:1106.2822 [hep-ex]].

[13] S. Fukasawa, M. Ghosh and O. Yasuda, Nucl. Phys. B 918 (2017) 337 doi:10.1016/j.nuclphysb.2017.02.008 [arXiv:1607.03758 [hep-ph]].

[14] P. Adamson et al. [MINOS Collaboration], Phys. Rev. Lett. 107 (2011) 181802 [arXiv:1108.0015 [hep-ex]].

[15] M. A. Acero et al. [NOvA Collaboration], arXiv:1806.00096 [hep-ex].

[16] Y. Abe et al. [DOUBLE-CHOOZ Collaboration], Phys. Rev. Lett. 108 (2012) 131801 [arXiv:1112.6353 [hep-ex]].

[17] F. P. An et al. [DAYA-BAY Collaboration], Phys. Rev. Lett. 108 (2012) 171803 [arXiv:1203.1669 [hep-ex]].

[18] J. K. Ahn et al. [RENO Collaboration], Phys. Rev. Lett. 108 (2012) 191802 [arXiv:1204.0626 [hep-ex]].

[19] F. Capozzi, E. Lisi, A. Marrone and A. Palazzo, Prog. Part. Nucl. Phys. 102 (2018) 48 [arXiv:1804.09678 [hep-ph]].

[20] P. F. de Salas, S. Gariazzo, O. Mena, C. A. Ternes and M. Tortola, arXiv:1806.11051 [hep-ph].

[21] K. Abe et al. [Hyper-Kamiokande Working Group], arXiv:1412.4673 [physics.ins-det].

[22] R. Acciarri et al. [DUNE Collaboration], arXiv:1512.06148 [physics.ins-det].

[23] K. Abe et al. [Hyper-Kamiokande Collaboration], PTEP 2018 (2018) no.6, $063 \mathrm{C} 01$ doi:10.1093/ptep/pty044 [arXiv:1611.06118 [hep-ex]].

[24] K. Abe et al., arXiv:1109.3262 [hep-ex].

[25] A. Gando et al. [KamLAND-Zen Collaboration], Phys. Rev. Lett. 117 (2016) no.8, 082503 Addendum: [Phys. Rev. Lett. 117 (2016) no.10, 109903] doi:10.1103/PhysRevLett.117.109903, 10.1103/PhysRevLett.117.082503 [arXiv:1605.02889 [hep-ex]].

[26] V. N. Aseev et al. [Troitsk Collaboration], Phys. Rev. D 84 (2011) 112003 doi:10.1103/PhysRevD.84.112003 [arXiv:1108.5034 [hep-ex]].

[27] A. Osipowicz et al. [KATRIN Collaboration], hep-ex/0109033.

[28] C. Yeche, N. Palanque-Delabrouille, J. Baur and H. du Mas des Bourboux, JCAP 1706 (2017) no.06, 047 doi:10.1088/1475-7516/2017/06/047 [arXiv:1702.03314 [astro-ph.CO]]. 
[29] Belle experiment, http://belle.kek.jp/.

[30] Babar experiment, http://www-public.slac.stanford.edu/babar/.

[31] A. Bandyopadhyay et al. [ISS Physics Working Group Collaboration], Rept. Prog. Phys. 72 (2009) 106201 [arXiv:0710.4947 [hep-ph]].

[32] K. N. Abazajian et al., arXiv:1204.5379 [hep-ph].

[33] L. Wolfenstein, Phys. Rev. D 17, 2369 (1978).

[34] M. M. Guzzo, A. Masiero and S. T. Petcov, Phys. Lett. B 260 (1991) 154.

[35] E. Roulet, Phys. Rev. D 44 (1991) 935.

[36] Y. Grossman, Phys. Lett. B 359 (1995) 141 doi:10.1016/0370-2693(95)01069-3 [hep-ph/9507344].

[37] S. Antusch, C. Biggio, E. Fernandez-Martinez, M. B. Gavela and J. Lopez-Pavon, JHEP 0610 (2006) 084 doi:10.1088/1126-6708/2006/10/084 [hep-ph/0607020].

[38] A. Aguilar et al. [LSND Collaboration], Phys. Rev. D 64 (2001) 112007 [arXiv:hep-ex/0104049].

[39] A. A. Aguilar-Arevalo et al. [MiniBooNE Collaboration], arXiv:1805.12028 [hep-ex].

[40] P. Adamson et al. [Daya Bay and MINOS Collaborations], Phys. Rev. Lett. 117 (2016) no.15, 151801 Addendum: [Phys. Rev. Lett. 117 (2016) no.20, 209901] doi:10.1103/PhysRevLett.117.151801, 10.1103/PhysRevLett.117.209901 [arXiv:1607.01177 [hep-ex]].

[41] N. Agafonova et al. [OPERA Collaboration], JHEP 1806 (2018) 151 doi:10.1007/JHEP06(2018)151 [arXiv:1803.11400 [hep-ex]].

[42] T. A. Mueller et al., Phys. Rev. C 83 (2011) 054615 [arXiv:1101.2663 [hep-ex]].

[43] P. Huber, Phys. Rev. C 84 (2011) 024617 Erratum: [Phys. Rev. C 85 (2012) 029901] doi:10.1103/PhysRevC.85.029901, 10.1103/PhysRevC.84.024617 [arXiv:1106.0687 [hep-ph]].

[44] G. Mention, M. Fechner, T. Lasserre, T. A. Mueller, D. Lhuillier, M. Cribier and A. Letourneau, Phys. Rev. D 83 (2011) 073006 [arXiv:1101.2755 [hep-ex]].

[45] C. Giunti and M. Laveder, Phys. Rev. D 77 (2008) 093002 [arXiv:0707.4593 [hep-ph]].

[46] M. C. Gonzalez-Garcia and M. Maltoni, JHEP 1309 (2013) 152 doi:10.1007/JHEP09(2013)152 [arXiv:1307.3092 [hep-ph]].

[47] M. Maltoni and A. Y. Smirnov, Eur. Phys. J. A 52 (2016) no.4, 87 doi:10.1140/epja/i2016-16087-0 [arXiv:1507.05287 [hep-ph]].

[48] S. Davidson, C. Pena-Garay, N. Rius and A. Santamaria, JHEP 0303 (2003) 011 [hep-ph/0302093].

[49] T. Ohlsson, Rept. Prog. Phys. 76 (2013) 044201 doi:10.1088/0034-4885/76/4/044201 [arXiv:1209.2710 [hep-ph]].

[50] O. G. Miranda and H. Nunokawa, New J. Phys. 17 (2015) no.9, 095002 doi:10.1088/1367-2630/17/9/095002 [arXiv:1505.06254 [hep-ph]].

[51] E. Fernandez-Martinez, J. Hernandez-Garcia and J. Lopez-Pavon, JHEP 1608 (2016) 033 doi:10.1007/JHEP08(2016)033 [arXiv:1605.08774 [hep-ph]]. 